\title{
Repensando 0 ato de educar nossos pacientes
}

\author{
Ana Elizabeth Prado Lima Figueiredo \\ Escuela de Ciencias de la Salud. Facultad de Enfermería Pontifícia Universidade Católica do Rio Grande do Sul \\ (PUCRS). Brasil
}

Como citar este artículo:

Figueiredo A. E. Repensando o ato de educar nossos pacientes. Enferm Nefrol. 2019 Abr-Jun;22(2):109-11

0 ato de educar pacientes faz parte do processo de trabalho do enfermeiro: assistir; no entanto quantos de nós nos sentimos adequadamente preparados para ensinar nossos pacientes para o autocuidado? Não tenho dúvidas, que sabemos o necessário e também o que deve ser ensinado aos pacientes, a pergunta é, estamos preparados para ensinar? Num primeiro momento a resposta que me vem é que não somos preparados para esta atividade. Neste contexto podemos ainda acrescentar que a atividade de treinamento/educação dos pacientes não é valorizada adequadamente e que muitos ainda acreditam que expertise vem com a prática, e que qualquer um que sabe fazer sabe ensinar, o que discordo completamente.

Alguns conceitos básicos são fundamentais para a nossa qualificação, o entendimento de que ensinar e aprender são diferentes, que as pessoas aprendem de maneiras diversa e que o nosso modo de aprender pode interferir no nosso modo de ensinar. Segundo Knowles ${ }^{1}$, os adultos aprendem baseado em seis princípios dentre eles: a motivação, as experiências passadas influenciam a aquisição de novos conhecimento, precisam de objetivos claros e relevância prática para o aprendizado e gostam de serem respeitados. 0 aprendizado se concretiza quando há algum tipo de mudança (conhecimento, atitude, hábitos, comportamento). No entanto, devemos considerar que nosso paciente além de adulto, na maioria das vezes, são portadores de doenças crônicas que podem ter diversas manifestações inclusive sintomas depressivos, atenção reduzida e diminuição do nível de concentração, necessitando muitas repetições ${ }^{2}$ tornando a atividade de ensinar um desafio.

Aspecto importante para que o processo ensino aprendizagem se concretize é o entendimento dos diversos estilos de aprendizagem e de que existem algumas barreiras que podem interferir neste processo. Limitação da audição, visão, letramento em saúde inadequado, conforto alterado e entendimento limitado do idioma em que se está ensinamento são algumas da mais comuns. Assim, como o paciente, nós enfermeiros, temos maneiras específicas de aprender e ensinar, devemos ter claro, no entanto, que nossa maneira de aprender nem sempre se aplica aos pacientes. Para melhoria do processo ensino aprendizagem a clareza de objetivos e conhecimento de como o paciente/cuidador percebe e processa a informação recebida, irá contribuir sobre maneira para a eficácia do treinamento. Existem várias definições para estilos de aprendizagem, esta pode ser superficial, profunda ou estratégica, ou a definição mais tradicional como visual, aural, ler e escrever e motora. Independente do estilo empregado para identificar o estilo de aprendizagem 0 educador deve ter objetivos claros do que quer ensinar. A taxonomia de Bloom pode ser usada com este propósito visto que determina nível de proficiência e competências que devem ser alcançados no domínio cognitivo, processos cognitivo e de conhecimento ${ }^{3}$.

Frente a todos os estudos sobre a temática, fica claro que este processo ensino aprendizagem não é fácil e nem simples. Recentemente Taylor et al. ${ }^{4}$, propuseram um modelo múltiplo que engloba outros já existentes, que considera o conhecimento já adquirido, e parte dele para a construção do novo, este parece promissor para ser aplicado ao ensino dos nossos paciente.

Finalizando, quanto mais lemos sobre o assunto, mais cientes ficamos que existem muitas maneiras de explicar como os adultos aprendem, mas nenhuma explica completamente todos os aspectos deste processo. Portanto, cabe a nós enfermeiros buscar maior entendimento de quem são nossos pacientes, quais suas barreiras para aprender e como podemos trespassá-las com sucesso. Importante lembrar sempre que o foco principal da educação do paciente renal crônico é habilitá-lo para gerenciar seu cuidado de maneira segura. Esta reflexão terá atingido seu objetivo se você ao final da leitura se perguntou: como eu aprendo? Como eu ensino? Como posso melhorar? 


\section{Repensando la acción de educar a nuestros pacientes}

La acción de educar pacientes forma parte del proceso del trabajo de la enfermera: cuidar; sin embargo ¿cuantos de nosotros nos sentimos adecuadamente preparados para enseñar a nuestros pacientes el autocuidado? No tengo dudas, que sabemos lo necesario y también lo que debemos enseñar a los pacientes; la pregunta es, ¿estamos preparados para enseñar? En un primer momento, la respuesta que me invade es que no estamos preparados para esta actividad. Sin embargo, en este contexto podemos añadir que la actividad de entrenamiento y educación de los pacientes no es valorada adecuadamente, y que muchos todavía creen que la experiencia viene con la práctica, y que cualquiera que sepa hacer, sabe enseñar, con lo que estoy completamente en desacuerdo.

Algunos conceptos básicos son fundamentales para nuestra cualificación, el entendimiento de que enseñar y aprender son diferentes, que las personas aprenden de maneras diferentes y que nuestro modo de aprender puede interferir en nuestro modo de enseñar. Según Knowles ${ }^{1}$, los adultos para aprender se basan en seis principios fundamentales, de ellos destacan: la motivación, las experiencias pasadas influyen en la adquisición de nuevos conocimientos, necesitan de objetivos claros y práctica relevante para el aprendizaje y les gusta ser respetados. El aprendizaje se concreta cuando hay algún tipo de cambio (conocimiento, actitud hábitos, comportamiento). No obstante, debemos considerar que nuestros pacientes además de adultos, en la mayoría de los casos, son portadores de enfermedades crónicas que pueden tener diversas manifestaciones incluyendo síntomas depresivos, atención reducida y disminución del nivel de concentración, necesitando de muchas repeticiones ${ }^{2}$, convirtiendo la actividad de enseñar un desafío.

Un aspecto importante para que el proceso de enseñanza y aprendizaje se materialice, es el entendimiento de los diferentes estilos de aprendizaje y de que existen algunas barreras que pueden interferir en este proceso. La limitación de la audición, visión, escasos conocimientos en materia de salud, confort alterado y comprensión limitada del idioma en que se está enseñando, son algunas de las más comunes. Las enfermeras tenemos maneras específicas de aprender y enseñar, debemos tener claro, no obstante, que nuestra manera de aprender no siempre se aplica a los pacientes. Para mejorar el proceso de enseñanza y aprendizaje, la claridad de los objetivos y conocimientos de como el paciente/cuidador percibe y procesa la información recibida, contribuirá a la eficacia del entrenamiento. Existen varias definiciones para estilos de aprendizaje, estos pueden ser superficial, profundo o estratégico, o la definición más tradicional como visual, auditivo, leer y escribir y motora. Independientemente del estilo utilizado para identificar el estilo de aprendizaje, el educador debe tener claros los objetivos de lo que va a enseñar. La taxonomía de Bloom puede ser utilizada con este propósito, una vez que se determine el nivel de pericia y competencia que deben ser alcanzados en el dominio cognitivo, procesos cognitivos y de conocimientos ${ }^{3}$.

Frente a todos los estudios sobre el tema, queda claro que este proceso de enseñanza y aprendizaje no es fácil ni simple. Recientemente Taylor el al. ${ }^{4}$, propusieron un modelo múltiple que engloba otros ya existentes, que considera el conocimiento adquirido y parte de él, para la construcción del nuevo. Esto parece prometedor para ser aplicado a la enseñanza de nuestros pacientes.

Para terminar, cuanto más leemos acerca del asunto, más conscientes somos que existen muchas maneras de explicar cómo los adultos aprenden, pero ninguna explica completamente todos los aspectos de este proceso. Por lo tanto, las enfermeras deben buscar una mayor comprensión de quienes son nuestros pacientes, cuáles son sus barreras para aprender y cómo podemos atravesarlas con éxito. Es importante recordar que el foco principal de la educación del paciente renal crónico es capacitarlo para gestionar su cuidado de manera segura. Esta reflexión habrá conseguido su objetivo si al final de este texto, el lector se preguntó: ¿Cómo aprendo?, ¿Cómo enseño? ¿Cómo puedo mejorar? 


\section{Bibliografía}

1. Knowles MS, Holton III EF, Swanson RA. The adult learner: The definitive classic in adult education and human resource development: Routledge; 2014.

2. Baer C. Principles of Patient education. In: Lancaster L, editor. Core Curriculum for Nephrology Nursing. 4th ed. Pitman, NJ: American Nephrology Nurses Assoc; 2001. p. 191-201.

3. Ferraz A, Belhot RV. Taxonomia de Bloom: revisão teórica e apresentação das adequações do instrumento para definição de objetivos instrucionais. Gest Prod, São Carlos. 2010;17(2):421-31.

4. Taylor DC, Hamdy H. Adult learning theories: implications for learning and teaching in medical education: AMEE Guide No. 83. Medical teacher. 2013;35(11):e1561-72.

Este artículo se distribuye bajo una Licencia Creative Commons Atribución-NoComercial 4.0 Internacional. https://creativecommons.org/licenses/by-nc/4.0/

\section{Open Access CC) (\$)}

